\title{
MicroRNAs-mRNAs Expression Profile and Their Potential Role in Malignant Transformation of Human Bronchial Epithelial Cells Induced by Cadmium
}

\author{
Qun Liu, Chanjiao Zheng, Huanyu Shen, Zhiheng Zhou, and Yixiong Lei \\ School of Public Health, Guangzhou Medical University, Guangzhou 511436, China \\ Correspondence should be addressed to Zhiheng Zhou; zhihengz@163.com and Yixiong Lei; gz-leizeng@163.com
}

Received 10 April 2015; Revised 1 June 2015; Accepted 9 June 2015

Academic Editor: Wilma Kempinas

Copyright (c) 2015 Qun Liu et al. This is an open access article distributed under the Creative Commons Attribution License, which permits unrestricted use, distribution, and reproduction in any medium, provided the original work is properly cited.

\begin{abstract}
Background. Our study was designed to elucidate whether there were miRNA and mRNA aberrantly expression profiles and potential role in malignant transformation of $16 \mathrm{HBE}$ induced by Cd. Methods. mRNA and miRNA expression profiles were determined in 35th Cd-induced $16 \mathrm{HBE}$ and untreated $16 \mathrm{HBE}$ by microarray. A series of bioinformatics analyses such as predicting targets, GO, KEGG were performed to find DEGs, coexpressing networks between miRNAs and mRNAs and its functions. Results. 498 DEGs were found. 8 Cd-responsive novel miRNAs predicted previously were identified, and 5 of them were downregulated. 214 target genes were predicted for the Cd-responsive miRNAs, many of which appeared to regulate gene networks. Target gene CCM2 was showed reciprocal effect by miRNAs. According to the combination analysis, hsa-miR-27b-3p regulated most of the mRNAs, especially upregulated expression genes. The differentially expressed miRNAs are involved in the biological processes and channels, and these GO and KEGG enrichment analyses result were significantly enriched in the Cd-responsive. Discussion. These results provided a tight link for the miRNA-mRNA integrated network and implied the role of novel miRNAs in malignant transformation of $16 \mathrm{HBE}$ induced by Cadmium. It is better to understand the novel molecular mechanism of cadmium-induced tumorigenesis.
\end{abstract}

\section{Introduction}

Cadmium $(\mathrm{Cd})$ is a heavy metal that is widely applied in industry. However, its toxicity affects human health through occupational and environmental exposure [1-3]. High levels of $\mathrm{Cd}$ can be accumulated in various organs, and toxicological responses to $\mathrm{Cd}$ exposure include lung, kidney, and liver damage [4-6]. Several lines of experimental evidence have shown that $\mathrm{Cd}$ is carcinogenic to human and animals [7]. Based on epidemiology and laboratory evidences, $\mathrm{Cd}$ and its compounds were classified as human carcinogens in 1993 by the International Agency for Research on Cancer (IARC) [8].

The molecular mechanism is well understood in the etiology of heavy metal-associated diseases, including known interactions with epigenetic changes, protein functional groups, DNA damage, increased DNA replication, and cell division [9-11]. Most of these associations have been demonstrated by mRNA expression assays $[12,13]$ and experimental data also have linked altered miRNAs expression with exposure Cd recently [11, 14].

Small RNAs may be involved in the regulation/signaling of metal toxicity response [14, 15]. miRNAs are a class of endogenous, noncoding, single-stranded, small RNA molecules of 19-22 nucleotides. miRNAs regulate expression of target genes at the posttranscriptional level by binding to $3^{\prime}$ untranslated regions of target mRNAs [16]. Moreover, the functional strand of mature miRNA loads onto the RNAinduced silencing complex (RISC) to suppress the expression of specific mRNA targets by directing mRNA degradation or translational repression [17, 18]. In addition, miRNAs also play a serious role in cell fate decisions; they are involved in the many vital biological events, including proliferation, differentiation, apoptosis, metabolism and viral infection, and many diseases including diseases of various organ systems, metabolic disorders, and cancer at several sites [19-21]. Many 
miRNAs have been proved to act as oncogenes and tumor suppressors [22].

miRNAs have widespread impact on expression and evolution of protein-coding genes [23]. To date, several computational methods have been successfully developed for predicting miRNAs and their targets $[24,25]$. With regard to the species-specific miRNAs, they can be predicted depending on the intragenomic matching between miRNA candidates and their targets coupled with support vector machine classification of miRNA precursors. Inhibition efficiency of miRNAs to target genes mainly was determined by the $5^{\prime}$ end of a nucleotide sequence and site from 2 to 8 .

The microarray analysis revealed that this metal modulated expression of nearly 400 genes in Arabidopsis and more than 1,700 in rice [26]. Recent research showed that cadmium stress affects the levels of miRNAs in soybean, rape plants, and rice [27-31]. Martínez-Pacheco et al. investigated the immortalized mouse fibroblast cell line BALB/3T3 A311-1 with exposed metal mixture including cadmium, which showed that the metal mixture resulted in miRNA expression profile that might be responsible for the mRNA expression changes observed under experimental conditions in which coding proteins were involved in cellular processes, including cell death, growth, and proliferation related to the metalassociated inflammatory response and cancer [32].

We previously established a model system of morphological cell transformation with cadmium chloride $\left(\mathrm{CdCl}_{2}\right)$ in $16 \mathrm{HBE}[12]$. This could provide in vitro human model system to examine the molecular events occurring of Cd carcinogenesis [12]. In the current study, Cd-transformed cells at the 35th passage were chosen as the treatment group and untreated normal $16 \mathrm{HBE}$ as the control group. In order to identify and study differentially expressed mRNAs and miRNAs after cadmium exposure, miRCURY LNA 7th miRNA chip and the Human mRNA Array v2.0 $(8 \times 60 \mathrm{~K}$, Arraystar $)$ were simultaneously used for high-throughput screening. And this can also provide theory evidence for further researching. After the related target genes were identified, bioinformatics methods of GO and pathway analysis were used.

\section{Materials and Methods}

2.1. Cell Culture and Treatments. 16HBE cells were morphologically transformed using $\mathrm{CdCl}_{2}$, as previously described [12]. Untransformed 16HBE cells (controls) and Cd-transformed cells at the 35 th $\left(5 \mu \mathrm{mol} \mathrm{L}{ }^{-1} \mathrm{Cd}\right.$ for 14 weeks) passage were cultured in RPMI-1640 containing L-glutamine (Sigma, St Louis, USA), 10\% fetal bovine serum (FBS), and 1\% penicillin/streptomycin (Life, California Carlsbad, USA) at $37^{\circ} \mathrm{C}$ in a $5 \% \mathrm{CO}_{2}$ humidified atmosphere. The cells were passaged twice weekly and cells in logarithmic growth phase $(2-5 \times$ $10^{5}$ cells $/ \mathrm{mL}$ ) were harvested for following experiments.

2.2. RNA Isolation. Total RNA was harvested by using TRIzol (Invitrogen, California Carlsbad, USA) and miRNeasy mini kit (QIAGEN, Dusseldorf, Germany) according to manufacturer's instructions. The RNA quality was assessed on agarose gels and the concentration was determined with a NanoDrop spectrophotometer (ND-1000, Namedrops Technologies, Wilmington, DE, USA).

2.3. mRNA Expression Assays and Analysis. Microarray assays were performed by a service provider, Kangchen Biotech (http://www.kangchen.com.cn/). The sample preparation and microarray hybridization were performed based on the manufacturer's standard protocols with minor modifications. Briefly, mRNA was purified from total RNA after removal of rRNA (mRNA-ONLY Eukaryotic mRNA Isolation Kit, Epicentre, Wisconsin Madison, USA). Then, each sample was amplified and transcribed into fluorescent cRNA along the entire length of the transcripts without $3^{\prime}$ bias utilizing a random priming method. The labeled $c$ RNAs were hybridized onto the Human mRNA Array v2.0 $(8 \times 60 \mathrm{~K}$, Arraystar, Shanghai, China). For our experiments, scanned images were then imported into Agilent Feature Extraction software (version 11.0.1.1) for grid alignment and data extraction. Experiments were conducted in three $16 \mathrm{HBE}$ and three 35 th $16 \mathrm{HBE}$, and the 35 th $16 \mathrm{HBE}$ were treated with different concentration of cadmium chloride (low toxicity, medium toxicity, and high toxicity).

Quantity normalization and subsequent data processing were performed by using the GeneSpring GX v11.5.1 software package (Agilent, California, USA). After quantile normalization of the raw data, mRNAs where at least 5 out of 6 samples had flags in present or marginal ("All Targets Value") were chosen for further data analysis. Differentially expressed mRNAs were identified through fold change (fold change $\geq$ $2.0)$ and $t$-test $(p$ values $<0.05)$ filtering.

2.4. miRNA Expression Assays and Analysis. MiRNA microarray assays were also performed by a service provider, Kangchen Bio-tech (http://www.kangchen.com.cn/). The 3,100 miRNA sequences were predicted by Lindow et al. The 7th generation of miRCURY LNA Array (v.18.0) (Exiqon, Copenhagen, Denmark) was used to identify Cd-responsive miRNAs on microarray chips. Microarray experiments were performed twice using distinct biological samples. Scanned images were then imported into GenePix Pro 6.0 software (Axon, Groningen, Holland) for grid alignment and data extraction. Replicated miRNAs were averaged and miRNAs with intensities $\geq 30$ in all samples were chosen for calculating normalization factor. Expressed data were normalized using the Median normalization. After normalization, differentially detected signals were those with fold change $\geq 1.3$ and $p$ values $<0.05$.

2.5. Bioinformatics Analysis of Data. Both mRNA and miRNA expression data were normalized with internal housekeeping sequences. The fluorescent signal intensity and background measurements were obtained on six spots for each mRNA. The results filtering and normalization were processed with the Microsoft Excel software, where the background value was subtracted from the fluorescence of the sample for both the control and experimental condition, and then compared between them to express the result regulation direction as fold change and $t$-test. Before comparing the differential expression of genes in response to Cd treatment, 
TABLE 1: Sequences of primers used in qRT-PCR detection.

\begin{tabular}{lc}
\hline Primer & Sequence \\
\hline has-miR-27b-3p & \\
$\begin{array}{l}\text { Forward } \\
\text { Reverse }\end{array}$ & $5^{\prime}$-ACACTCCAGCTGGG TTCACAGTGGCTAAG-3' \\
RT primer & $5^{\prime}$-ATCCAGTGCAGGGTCCGAGG-3' \\
\hline has-miR-944 & CTCAACTGGTGTCGTGGAGTCGGCAATTCAGTTGAG GCAGAACT \\
Forward & $5^{\prime}$-GCGGCGGAAATTATTGTACATC-3' \\
Reverse & $5^{\prime}$-ATCCAGTGCAGGGTCCGAGG-3' \\
RT primer & GTCGTATCCAGTGCAGGGTCCGAGGTATTCGCACTGGATACGACCTCATC \\
\hline CCM2 & \\
Forward & $5^{\prime}$-GAGACCATTGGCGTGAAGGA-3' \\
Reverse & $5^{\prime}$-GATGTCCGAGATCATGCGGT-3 \\
\hline
\end{tabular}

normalized gene expression levels were obtained by normalizing the number of raw clean tags in each library to the number of transcripts per million clean tags (TPM). A rigorous algorithm method was performed for the differential expression detection of genes across samples.

A combination of FDR $<0.001$ (using Benjamini and Hochberg method; 1995) and the absolute value of $\log _{2}$ Ratio $\geq 1$ was used as the threshold to determine the significance of differentially expressed genes. Online databases like miRanda, TargetScan, and miRBase were chosen and searched to find predicted targets. In order to further identify differential miRNA genes, which may play potential roles in biological processes, the differentially expressed targets of specific miRNA were compared with the differentially expressed mRNA, and the intersection genes were defined as relevantly potential genes and involved in potential pathways. GO and pathway enrichment analysis were based on the gene ontology (http://www.geneontology.org/) and KEGG pathways (http://www.genome.jp/kegg/) [33-35].

2.6. Quantitation Real-Time RT-PCR of miRNAs and Target Gene. Total RNA $(1 \mu \mathrm{g})$ was isolated from untreated $16 \mathrm{HBE}$ cells, 35th Cd-induced 16HBE cells, and tumorigenic cells using TRIzol according to manufacturer's instructions. The purity and integrity of total RNA were analyzed by spectrophotometry and agarose gel electrophoresis, respectively. After purification, the miRNAs were reverse transcribed into cDNA with the specific RT primer by the Prime Script RT reagent Kit (Promega, China). qPCR was performed using PCR reagent Kit (Promega, China) according to manufacturer's instructions. The expression of CCM2 mRNA was detected with reverse transcription-polymerase chain reactions (RT-PCRs) using Reverse Transcription System (Promega: Madison, WI, USA). Gene expression levels were calculated based on the comparative quantitative method (the $\Delta \Delta C T$ method) [36]. The primers used in qRT-PCR were listed in Table 1.

\section{Results}

3.1. Identification of Cd-Responsive Novel Candidate mRNAs. With abundant and varied probes $(30,215$ mRNAs) in our microarray, of them, there were $366 \mathrm{mRNAs}$ with upregulated expression and 132 with downregulated expression $(\geq 2.0$ fold change, $p \leq 0.05$; see Supplementary Table S1 in the Supplementary Material available online at http://dx.doi.org/10.1155/ 2015/902025) in Cd-induced 35th cells when compared with untreated 16HBE cells. The Volcano Plot (Figure 1(a)) was applied to identify differentially expressed miRNAs with statistical significance. The heat map diagram (Figure 1(b), Supplementary Table S2) showed the result of the two-way hierarchical clustering of mRNAs and samples.

3.2. Identification of Cd-Responsive Novel Candidate miRNAs. To identify differentially expressed miRNAs with statistical significance, we performed Volcano Plot (Figure 2(a)) filtering between the two groups from the experiment. miRNAs with intensities $\geq 30$ in all samples were chosen for calculating Median normalization factor. The threshold we used to screen up- or downregulated miRNAs is fold change $\geq 1.3$ and $p$ value $\leq 0.05$. A total of 8 putative miRNAs (Supplementary Table S3) were detected to be Cd-responsive by microarray assays. They were considered to be new miRNAs and named according to the probe ID on the microarray chips as follows: hsa-miR-27b-3p, hsvl-miR-H6-5p, hsa-miR-1265, hsa-miR944, hsa-miR-3960, hsa-miR-4708-3p, hsa-miR-877-5p, and hsa-miR-1261 (Table 2, Supplementary Table S5). hsa-miR27b-3p, hsvl-miR-H6-5p, and hsa-miR-1265 were upregulated under $\mathrm{Cd}$ stress, whereas the other miRNAs were all downregulated. The heat map diagram (Figure 2(b), Supplementary Table S4) showed hsa-miR-27b-3p, hsv1-miR-H6$5 \mathrm{p}$, and hsa-miR-1265 genes in Cd-induced 35th cells were higher (deeper) than in untreated $16 \mathrm{HBE}$ cells, suggesting that these differentially expressed genes in Cd-induced 35th cells were upregulated and this was consistent with the fact that 3 genes out of 8 differentially expressed genes were upregulated by differentially expressed genes analysis ( $y$-axis and $x$-axis).

3.3. Analysis of MicroRNAs to Target $m R N A$ s. In our further analysis, we focused on the trend of expression changes of miRNA and its target genes. In order to identify correlations between them, we searched three online databases to find predicted targets in the miRanda (http://www.microrna.org/), 
TABLE 2: Predicted targets of Cd-responsive miRNAs in human bronchial epithelial cells

\begin{tabular}{ll}
\hline miRNAs & Target genes \\
\hline $\begin{array}{l}\text { Upregulated } \\
\text { hsa-miR-27b-3p }\end{array}$ & CCM2, ADAMTS10, B4GALT3, C12orf35, DLGAP3, EML1, FAM108C1, GALNT5, \\
hsa-miR-1265 & ABRMAD2, KIAA0146... ELAVL2, IKZF4, MAGIX, PPAP2B, XPNPEP2... \\
$\begin{array}{l}\text { Downregulated } \\
\text { hsa-miR-877-5p }\end{array}$ & CCM2, ARFIP2, CAP1, FXR2, HSD11B1, PHF8, SMG5, SORBS3, TRIM10... \\
hsa-miR-944 & CCM2, AASS, BRIP1, C18orf34, DDX5, EPC2, FANCE, GAS6, HIVEP1, KCNH2, \\
hsa-miR-1261 & ZMYM6... \\
hsa-miR-3960 & AQP4, BRCA1, CCDC85C, EIF2A, EIF4E3, EIF4G2, FRMPD4, SUPT7L... \\
hsa-miR-4708-3p & C14orf43, HOXB8, MARVELD1, PCDHA8, PCDHA12, SLC9A3... \\
\hline
\end{tabular}

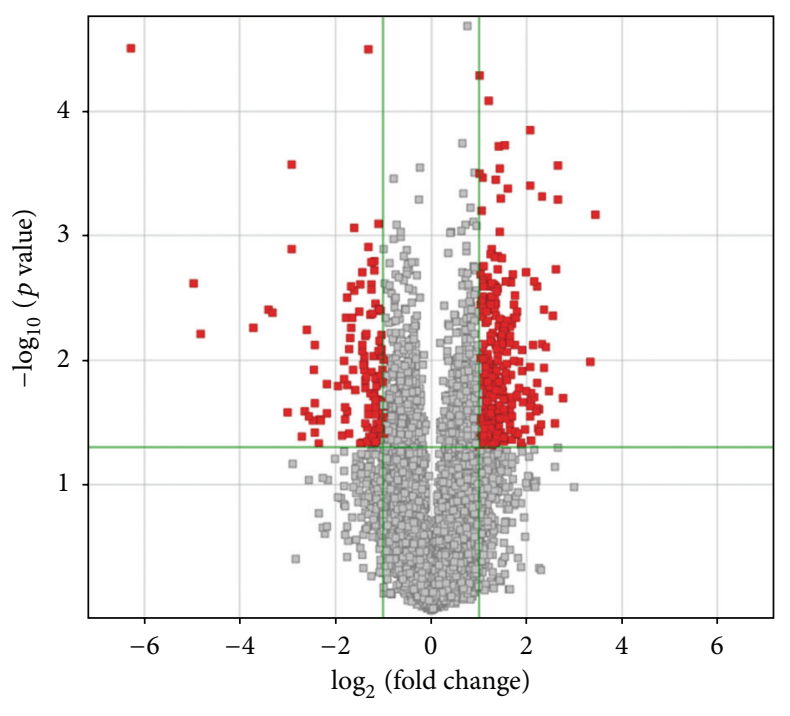

(a)

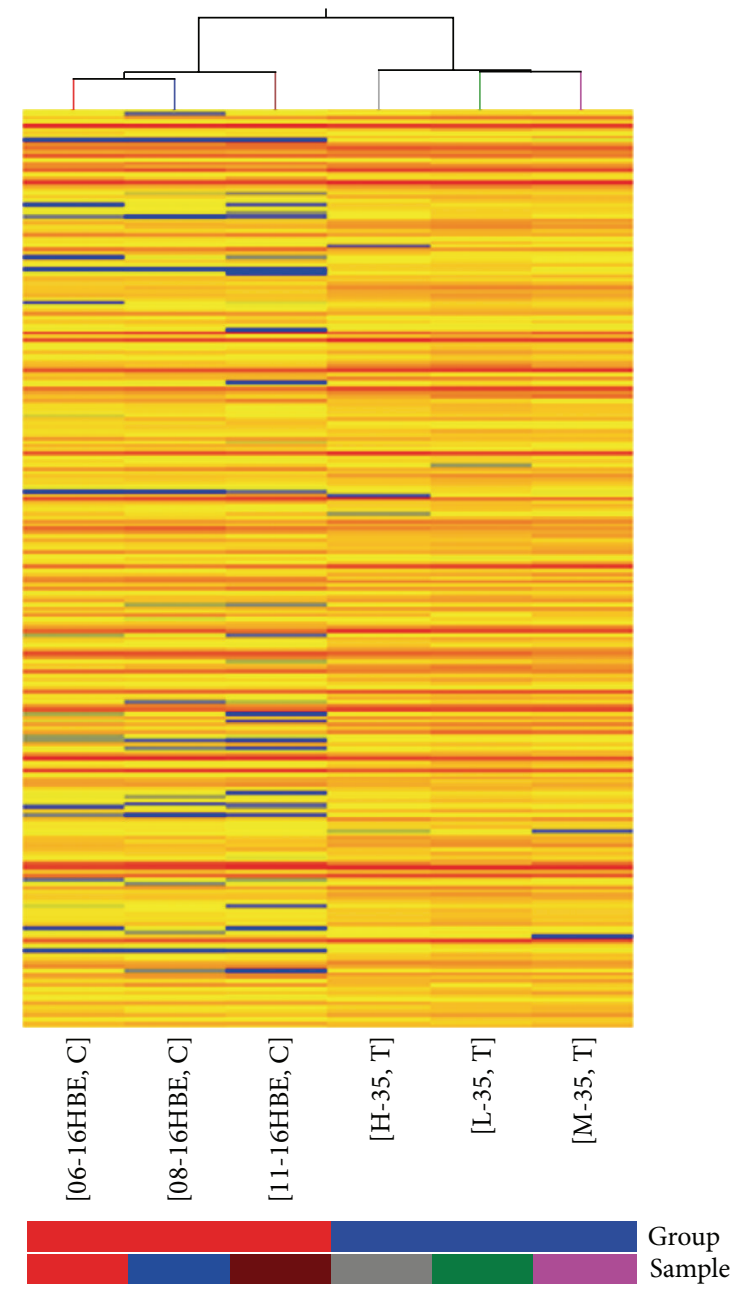

(b)

FIGURE 1: Differentially expressed mRNAs analysis. Volcano Plot (a) shows the relationship between fold change and statistical significance. The red points in the plot represent the differentially expressed mRNAs with statistical significance. The heat map diagram (b) shows cluster analysis of DEGs. The colors represents the expression values of DEGs. Sample names are listed in the horizontal axis, and high, medium, and low are $16 \mathrm{HBE}$ treated with different concentration of $\mathrm{CdCl}_{2}$ for 35 passaged. Right vertical axis represents the clustering of mRNAs. 


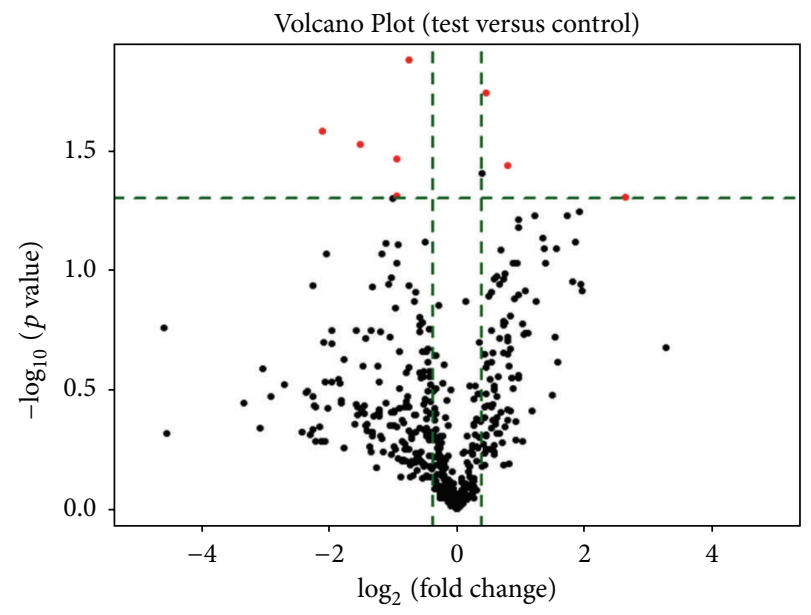

(a)
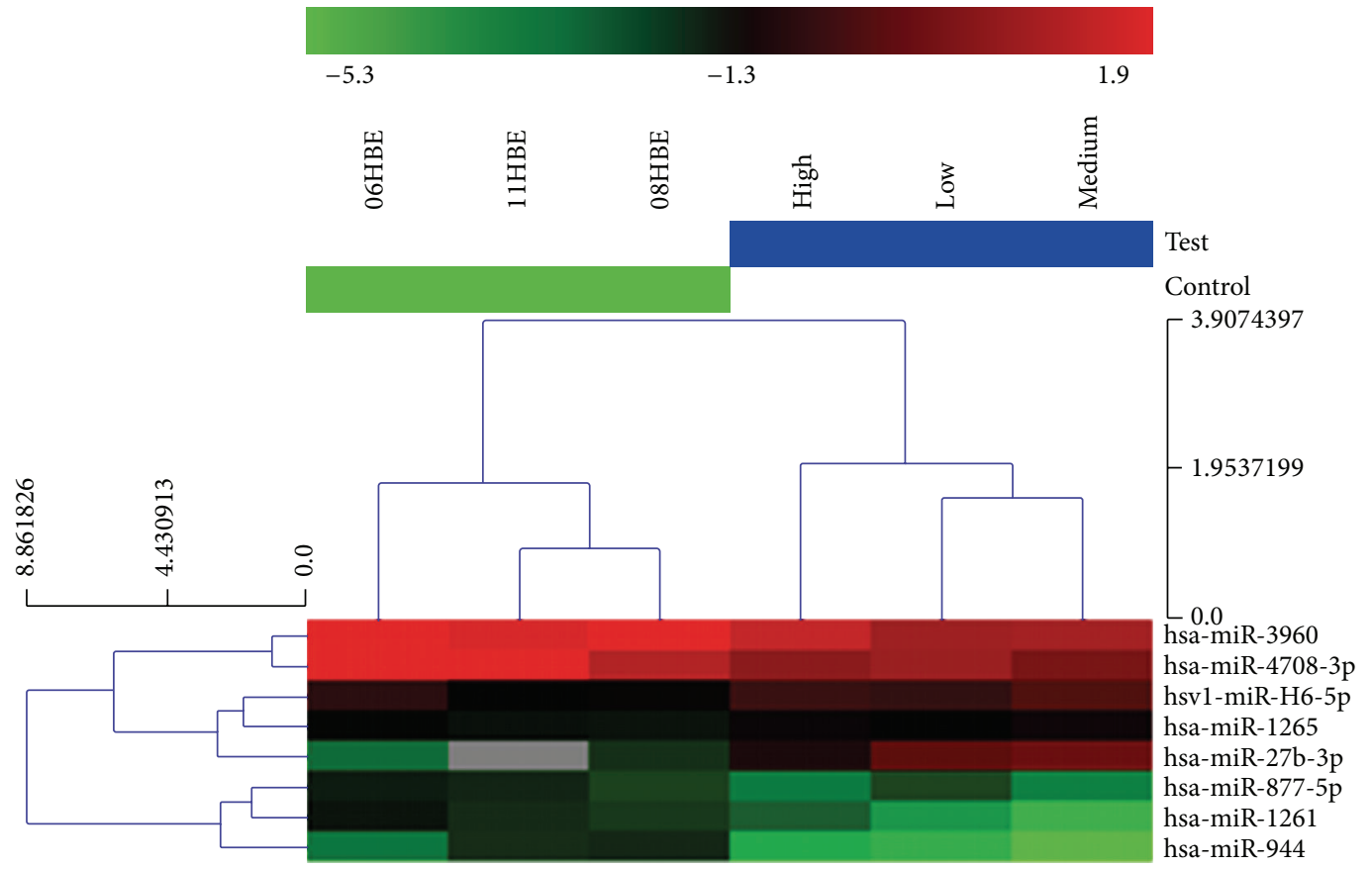

(b)

Figure 2: Differentially expressed miRNAs analysis. (a) Showing the relationship between fold change and statistical significance. The red points in the plot represent the differentially expressed miRNAs with statistical significance. (b) Showing cluster analysis of DEGs. The colors from green to red represent the fact that the expression values of DEGs become higher and higher. Sample names are listed in the horizontal axis, and high, medium, and low are $16 \mathrm{HBE}$ treated with different concentration of $\mathrm{CdCl}_{2}$ for 35 passaged. Right vertical axis represents the clustering of miRNAs.

TargetScan (http://www.targetscan.org/), and miRBase (http:// www.mirbase.org/). There were 17,259 miRNA-mRNA pairs in miRanda, 2,305 pairs in miRBase, and 2,752 pairs in TargetScan (Supplementary Table S5). A Venn diagram (Figure 3(a), Supplementary Table S5) showed that 214 of the microRNA-mRNA interaction pairs were acquired from three of the databases. The network figure (Figure 3(b)) showed the network relationship between miRNA and mRNA. CCM2 is the common target between hsa-miR-27b$3 \mathrm{p}$ and hsa-miR-944.
3.4. Combination Analysis of MicroRNA Microarray and $m R N A$ Microarray. According to the combination analysis, three miRNAs were found in the intersection, including hsamiR-27b-3p, hsa-miR-944, and has-miR-877-5p. Only the two former miRNAs could regulate same genes in mRNA microarray, especially has-miR-27b-3p. The details of genes were shown in Table 3.

3.5. GO Analysis of DEGs. For GO analysis, the ten most related genes and pathways were used. As shown by Figure 4 


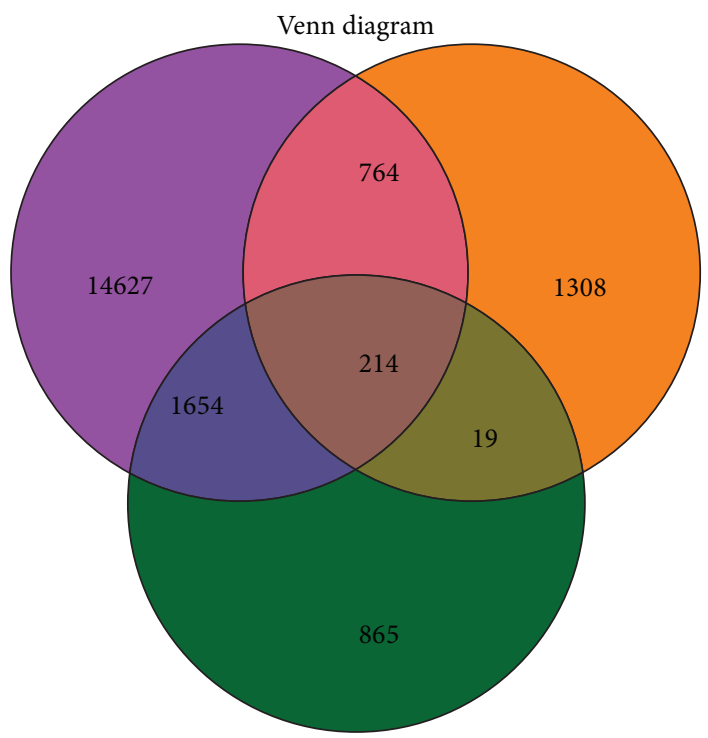

$$
\begin{aligned}
& \square \text { miRanda } \\
& \square \text { miRBase } \\
& \square \text { TargetScan }
\end{aligned}
$$

(a)
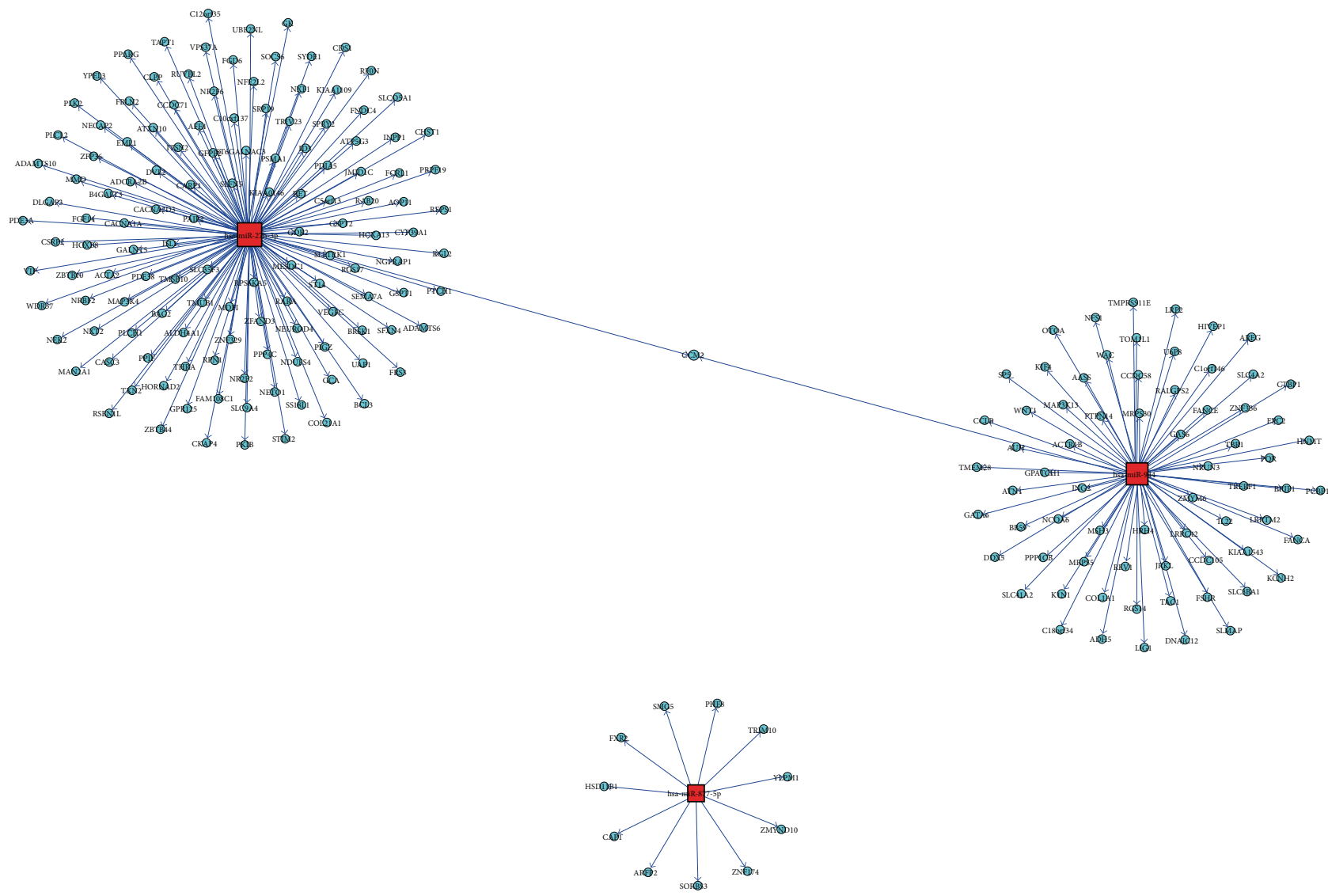

(b)

FIGURE 3: Targets of DEGs. (a) Venn diagrams showing the unique and shared regulated targets in DEGs; (b) showing the networks of 214 microRNA-mRNA interaction pairs of three of the databases. 


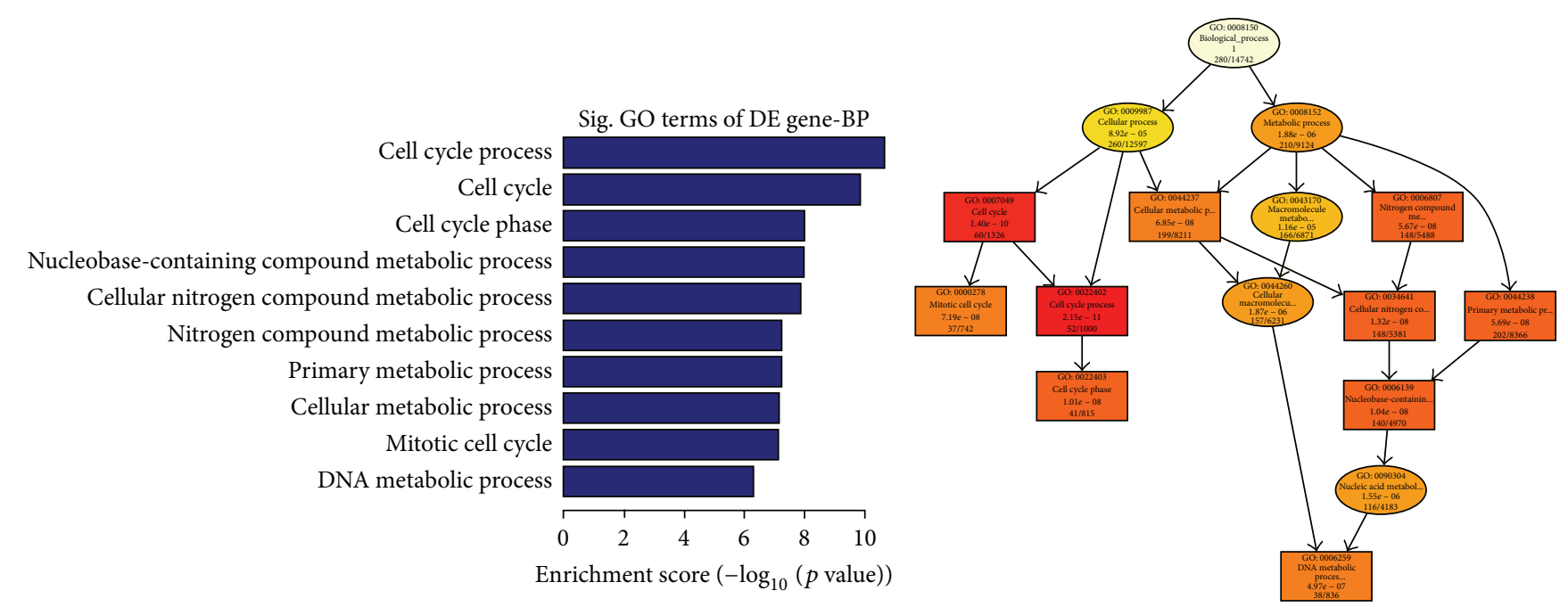

(a)

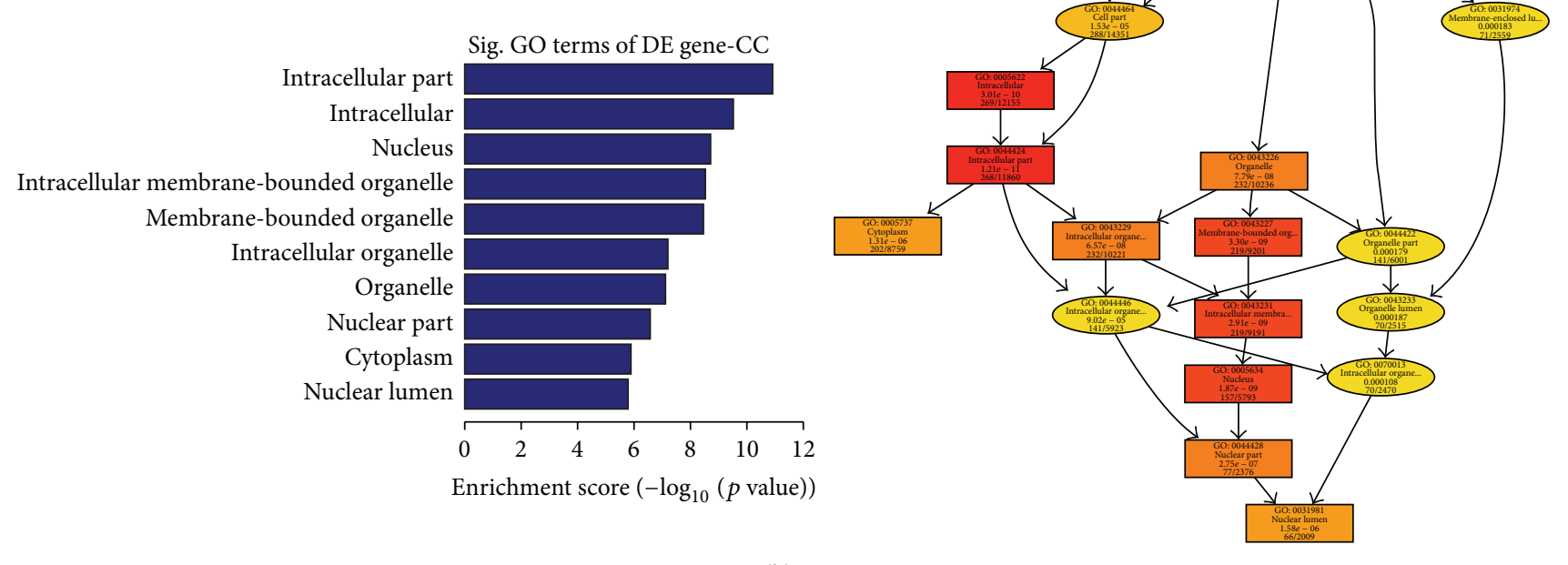

(b)

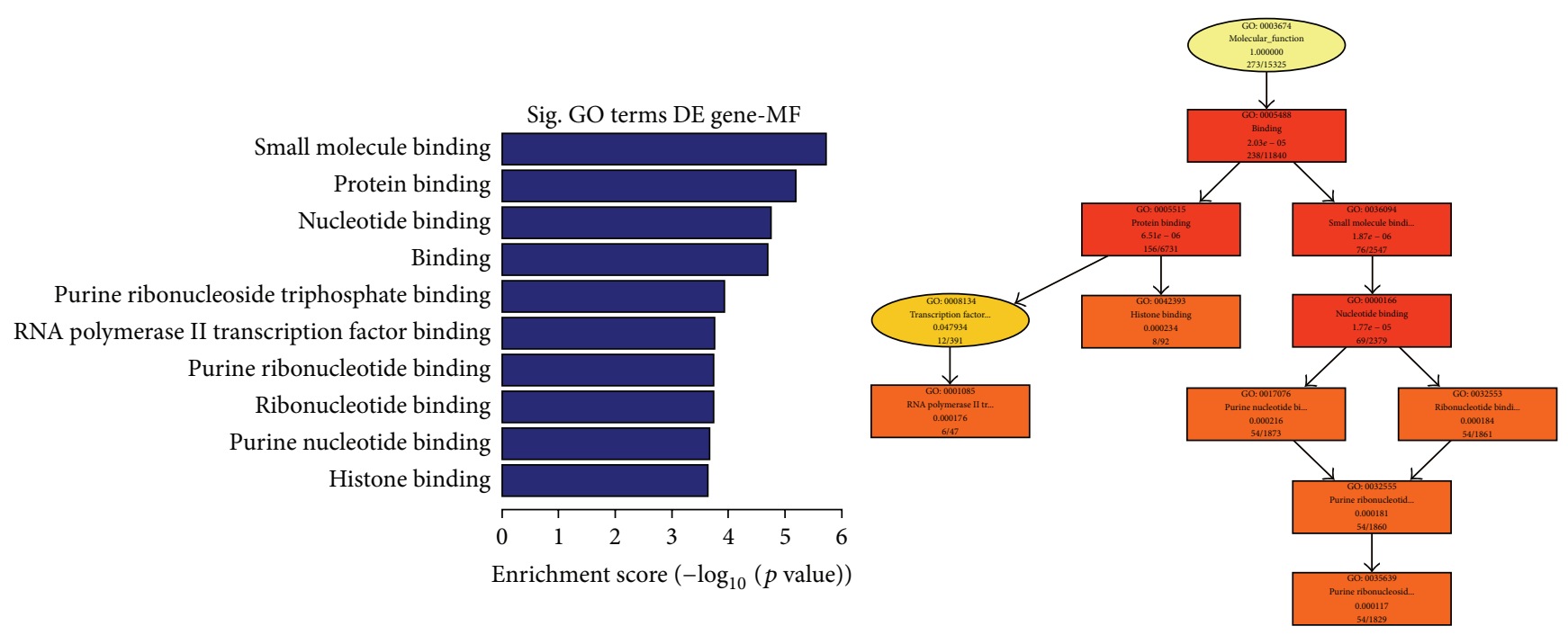

(c)

FIGURE 4: Continued. 

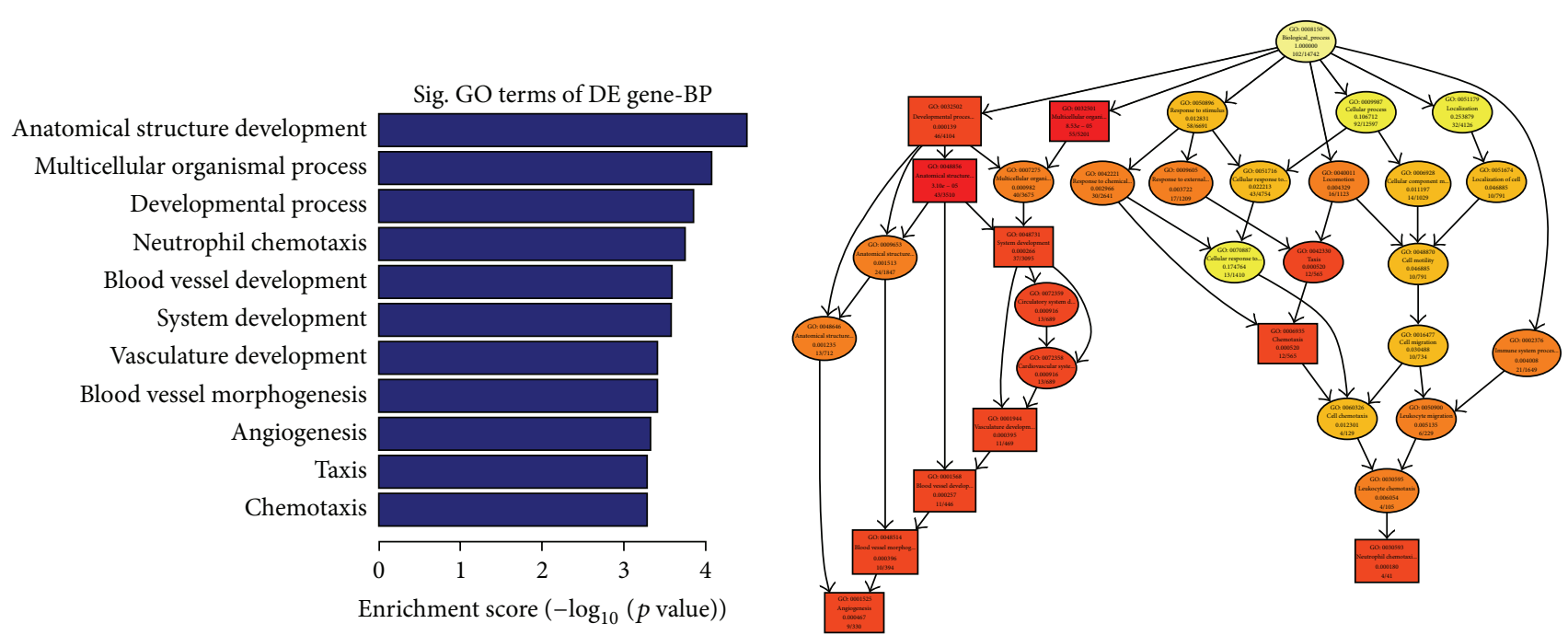

(d)
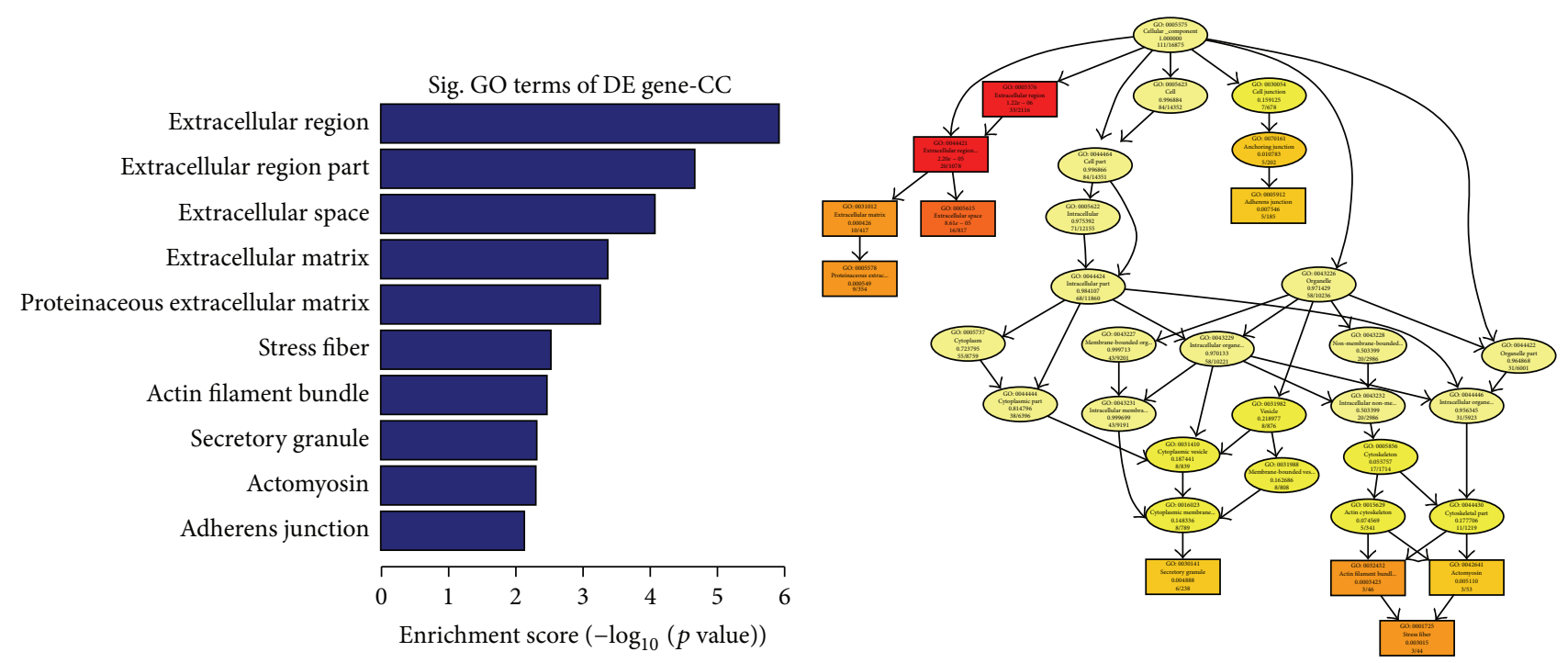

(e)
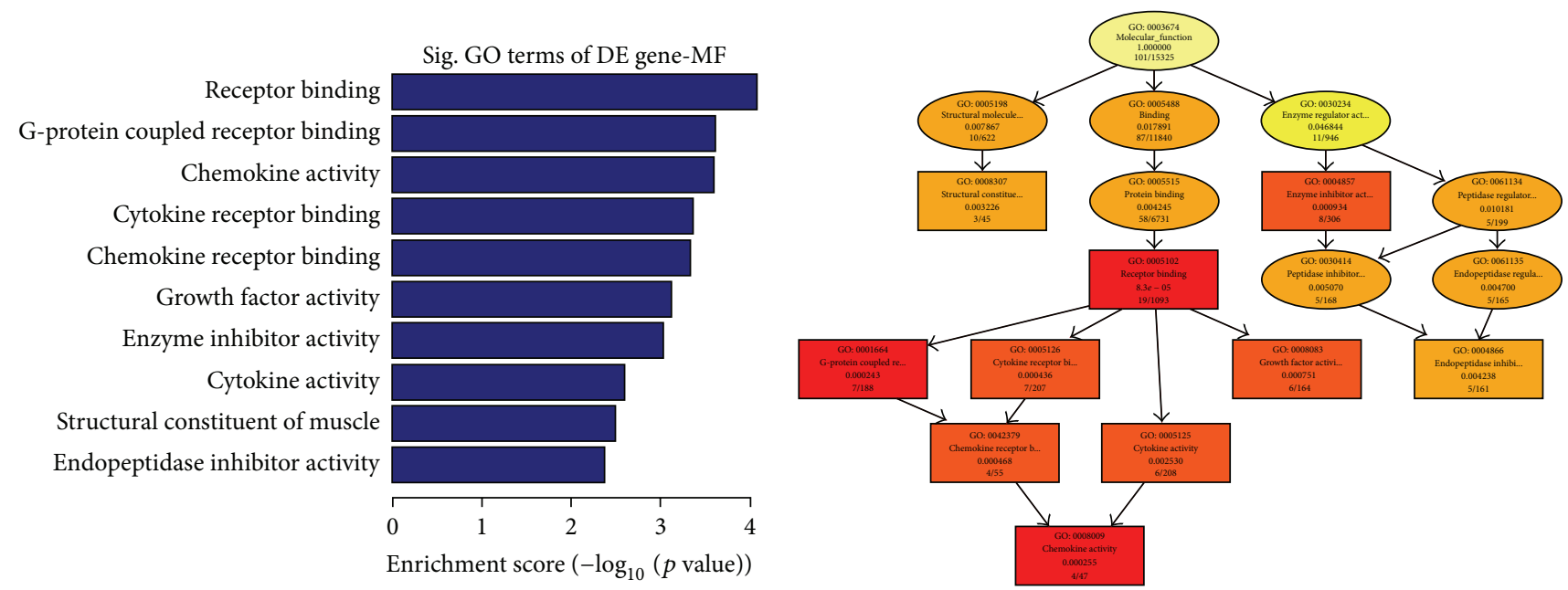

(f)

FiguRE 4: GO analysis of differentially expressed mRNAs in response to Cd stress. The top 10 of the most related parts were shown (a-f); $(a-c)$ showed biological process (a), cellular component (b), and molecular function (c) of upregulated mRNAs, respectively. (d-f) showed biological process (d), cellular component (e), and molecular function (f) of downregulated mRNAs. 
TABLE 3: Combinational analysis in the data of microRNA and mRNA microarray.

\begin{tabular}{|c|c|c|}
\hline & hsa-miR-27b-3p & hsa-miR-944 \\
\hline Up/down & Up & Down \\
\hline \multicolumn{3}{|l|}{ mRNAs } \\
\hline Upregulated & RGS17, ITSN2, PRPF19, and PLK2 & PPP1CB \\
\hline Downregulated & SYDE1 & DNAJC12 \\
\hline
\end{tabular}

(Supplementary Table S6), GO analysis showed that the aberrant mRNA expression genes took part in many biological processes: primary metabolism, cell metabolism, cell cycle progression, DNA damage and repair, biological cycle, and so forth. GO analysis showed that the targets of aberrant miRNA took part in many biological processes, such as primary metabolism, cellular response to stress, negative regulation of transcription, DNA-dependent, and regulation of endocrine process (Figure 5, Supplementary Table S7). These results supported the idea that Cd-induced malignant transformation was related to DNA damage and repair and biological cycle.

3.6. Pathway Analysis of DEGs. For the KEGG pathway analysis, pathways with $p$ value less than 0.05 were used. Pathway analysis of differentially expressed mRNAs (Figure 6, Supplementary Table S8) showed that they were involved in the cell cycle, P53 signaling pathway, prostate cancer, thyroid cancer, and endometrial cancer, Wnt signaling pathway, glioma, bladder cancer, pancreatic cancer, and axon guidance.

3.7. Expression Analysis of has-miR-27b-3p, has-miR-944, and Their Common Target Gene in Cd-Induced 16HBE Cells. A significant upregulated expression of has-miR-27b-3p and CCM2 in malignant 16HBE cells (16HBE cells, 35th Cdinduced $16 \mathrm{HBE}$ cells, and tumorigenic cells) was found, while has-miR-944 was downregulated in malignant $16 \mathrm{HBE}$ cells. The trend was aggravated as the cells passage (Figure 7). The results are consistent with microarrays.

\section{Discussion}

At present, lots of studies have confirmed molecular mechanisms of cadmium carcinogenesis including inhibit apoptosis, DNA repair and DNA methylation, histone acetylation $[9,11,37]$, reactive oxygen species in cadmium response, and cadmium-induced signal transduction pathways [38]. In recent years, microRNAs have become the research focus in the scientific field. It is identified that about $50 \%$ of human microRNA genes are located at fragile sites and genomic regions involved in cancers [39], which clarifies the important role of miRNA in the tumorigenesis. Global expression profiling analysis of miRNAs and mRNAs in the same samples may provide a unique opportunity to enhance our understanding of potential miRNA regulatory mechanisms in 16HBE cells which were exposed to Cd. In this study, these altered expression profiles of mRNA and miRNA were screened by mRNA and miRNA microarray after $16 \mathrm{HBE}$ cell exposed cadmium.
To further validate the regulatory role of miRNAs in Cdresponsive cells, the corresponding target mRNAs of Cdregulated miRNAs were predicted by intersection of the database of miRanda, TargetScan and miRBase. 214 target mRNAs of 3 miRNAs were identified by bioinformatics prediction (Table 1, Supplementary Table S5). 133 genes were found as the target genes of hsa-miR-27b-3p and 11 genes were found as the target genes of hsa-miR-877-5p; however 70 genes were found as the target genes of hsa-miR-944. CCM2 was the same target gene of hsa-miR-27b-3p and hsa-miR944. We also found that other miRNAs were related with CCM2 in these databases, miRanda, TargetScan, and miRBase. However the target genes of two miRNAs in TargetScan are CCM2, while more than two miRNAs' target genes are CCM2 in the nother two databases, both of them including hsa-miR-27b-3p, hsa-miR-877-5p, and hsa-miR-944 (Supplementary Table S5). The combination analysis could narrow down the range of miRNA candidates and clearly explicit the biological functions of target genes.

Most of miRNAs are clustered and shared similar expression patterns, implying that they are transcribed as polycistronic transcripts [40, 41]. Further research found the mutations of CCM2 gene lead to cerebral cavernous malformations. CCM2 encodes a scaffold protein that functions in the stress-activated p38 mitogen-activated protein kinase (MAPK) signaling cascade. The protein interacts with SMAD (small mothers against decapentaplegic) specific E3 ubiquitin protein ligase 1 (also known as SMURF1) via a phosphotyrosine binding domain to promote RhoA degradation. The protein is required for normal cytoskeletal structure, cellcell interactions, and lumen formation in endothelial cells. Multiple transcript variants encoding different isoforms have been found for this gene (provided by RefSeq, Nov 2009). The analysis of GO showed differently expressed miRNAs of Cd-responsive cells were reported to be involved in several biological processes, including negative regulation of transcription from RNA polymerase II promoter, cellular macromolecule metabolic process, and cellular response to stress, involved in several molecular functions transcription factor binding, ligand-activated sequence-specific DNA binding RNA polymerase II transcription factor activity, and protein binding. Those illustrated miRNAs are transcribed by RNA polymerase II as primary miRNAs (pri-miRNAs), which is consistent with the research of Cai et al., 2004 [42], Lee et al., 2004 [43], and so forth.

It is shown that cadmium can upregulate genes of encoding pathogen related proteins, antioxidant enzymes, transporters, TFs, and proteins associated with glutathione metabolism. With the literature supporting and confirmation, we show the importance of miRNAs in key cellular processes by modulating a small gene set in this study. Together, our findings allow us to assert the great importance of miRNAs in the development and/or establishment of cadmiumassociated diseases, especially cancer, through key cellular processes modulated by cadmium exposure targets that have also been cited as above. The patterns of mRNA and miRNA expression that predict establishment of cadmium health effects are helpful for understanding the aberrant molecular mechanisms of human cells. However, further study is 


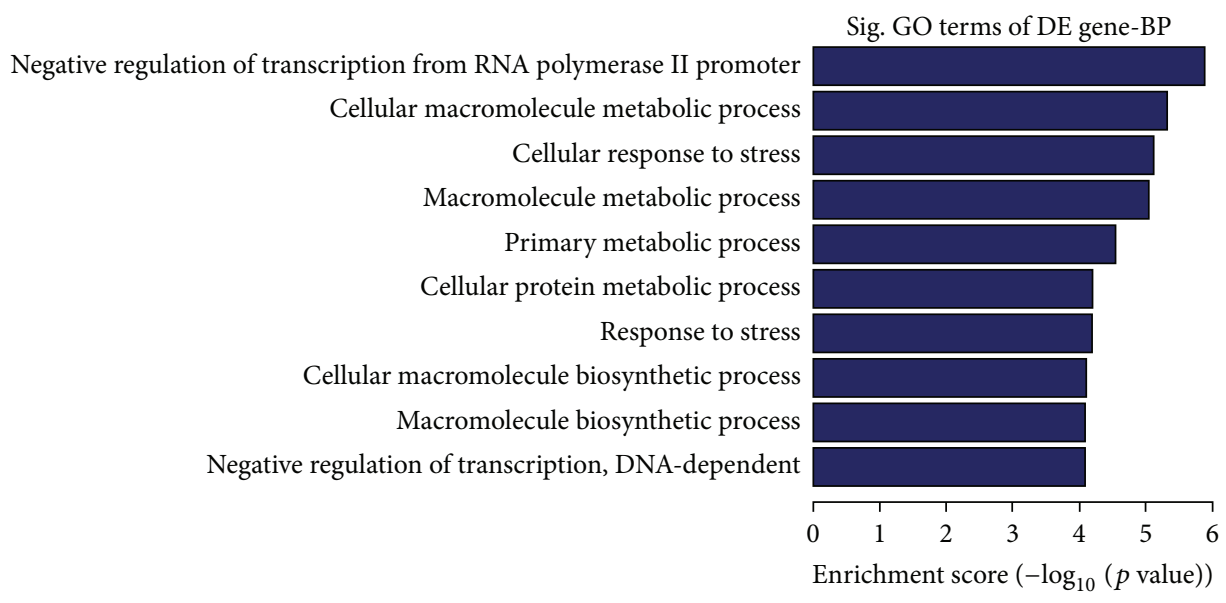

(a)

Sig. GO terms of DE gene-MF

Transcription factor binding

Sequence-specific DNA binding RNA polymerase II transcription factor activity

Protein binding

Steroid hormone receptor activity

Transcription regulatory region DNA binding

Regulatory region nucleic acid binding

Regulatory region DNA binding

Protein domain specific binding

Estrogen receptor binding

$3^{\prime}, 5^{\prime}$-cyclic-AMP phosphodiesterase activity Aldehyde dehydrogenase (NAD) activity

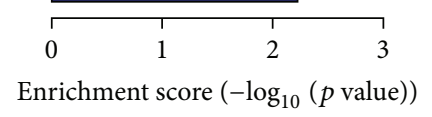

(b)

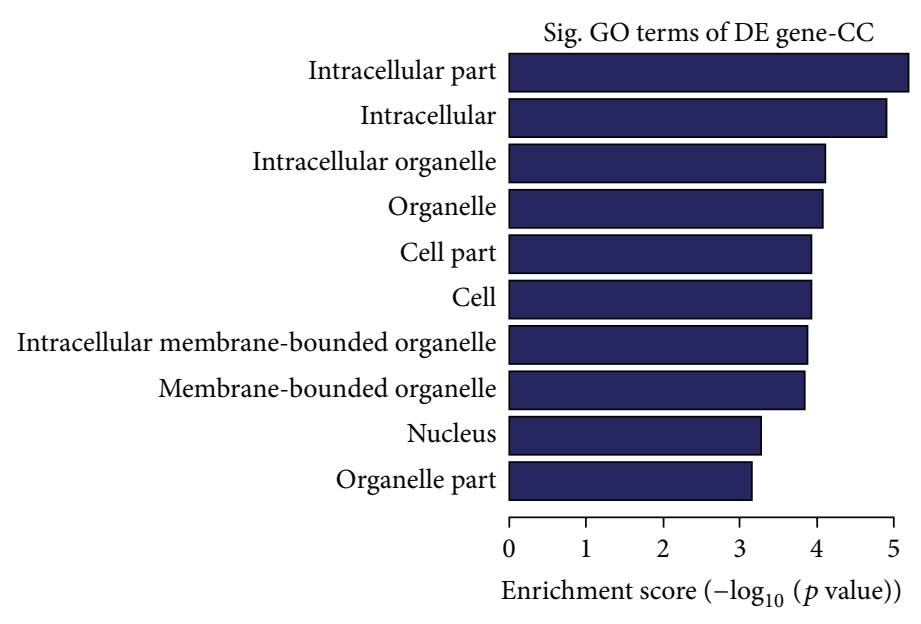

(c)

FIGURE 5: GO analysis of differentially expressed miRNA targets in response to Cd stress. The top 10 of the most related parts were shown $(\mathrm{a}-\mathrm{c})$; biological process (a), cellular component (b), and molecular function (c). 


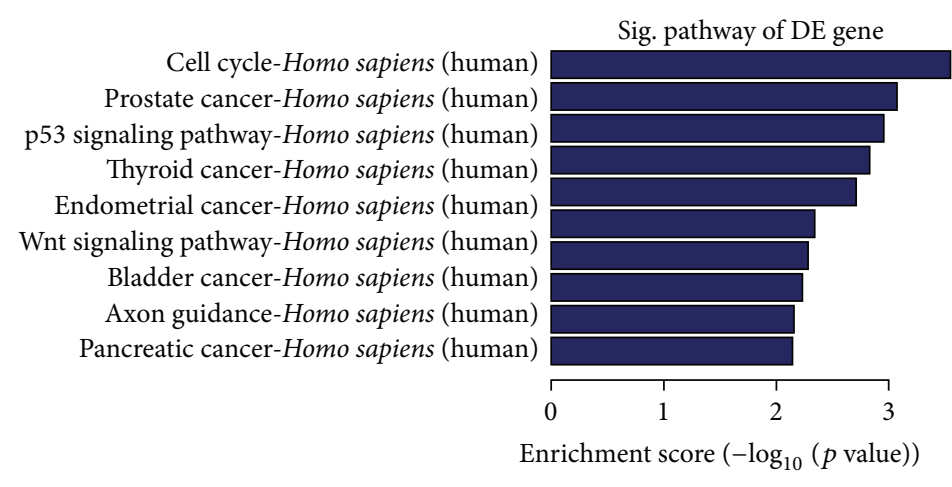

(a)

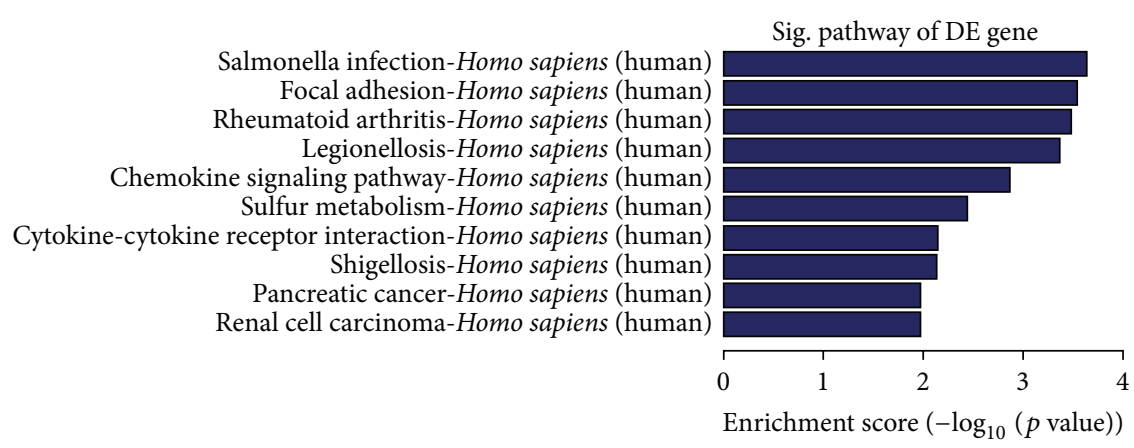

(b)

FIgURE 6: The KEGG pathway analysis of differentially expressed mRNAs. Pathways with $p$ value less than 0.05 were shown in the figure. All the $p$ values were transformed into $\log p$ value.

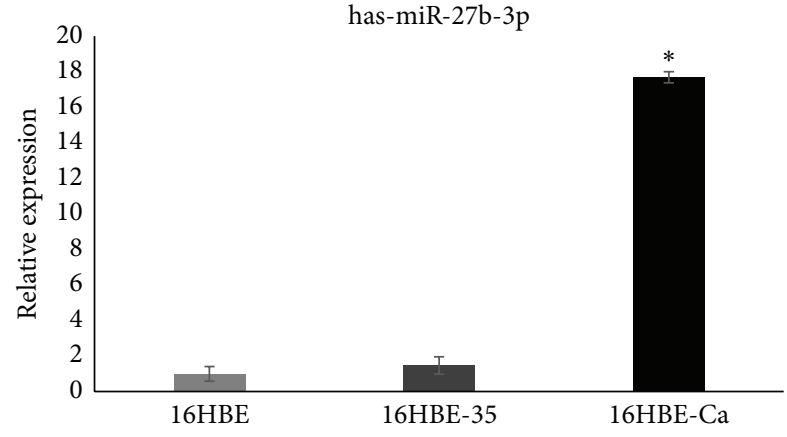

(a)

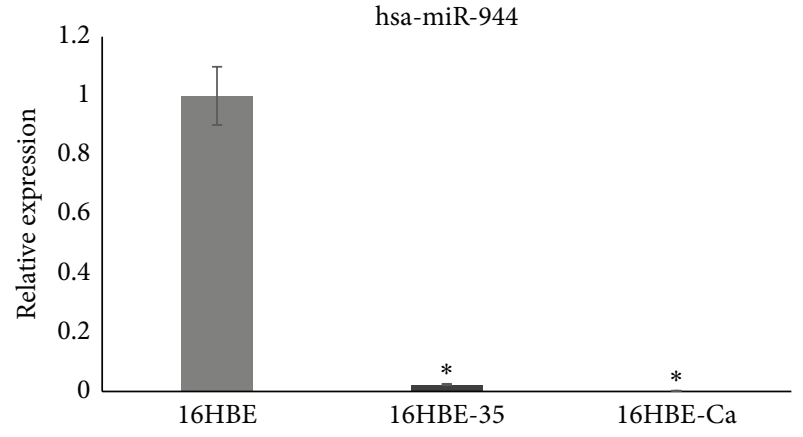

(b)

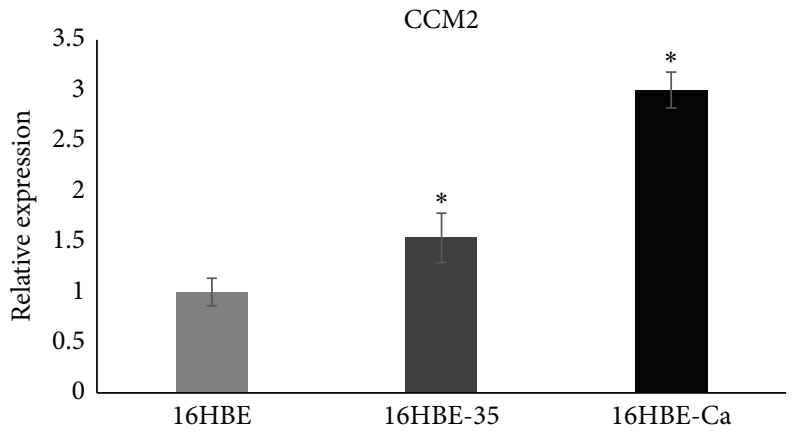

(c)

Figure 7: The expression of has-miR-27b-3p, has-miR-944, and CCM2 by qRT-PCR in different groups. $*$ represents $p<0.05$ as compared to $16 \mathrm{HBE}$, respectively. 
needed to understand the relationship between genetic predisposition and epigenetic disruption in the development of cadmium-associated diseases.

In summary, our results provide a tight link for the miRNA-mRNA integrated network after 16HBE exposed Cd. These results provide some new and meaningful candidate miRNAs and genes to allow further investigation of the mechanism after $16 \mathrm{HBE}$ differentiation and to provide several important molecular markers for the diagnosis and therapy of related diseases. Further study of the potential function of miRNA-mRNA integration in Cd-response will promote our understanding of the key role of $16 \mathrm{HBE}$ in Cd-response, such as further research of differentially expressed genes demonstrated by Western Blot, or various methods about the target gene CCM2 and genes of combination analysis.

\author{
Abbreviations \\ 16HBE: Human bronchial epithelial cells \\ Cd: Cadmium \\ $\mathrm{CdCl}_{2}$ : Cadmium chloride \\ $\mathrm{CO}_{2}: \quad$ Carbon dioxide \\ CIP: Calf Intestine Phosphatase \\ CCM2: Cerebral cavernous malformations 2 \\ DEGs: Differently expression genes \\ FDR: The false discovery rate \\ GO: Gene ontology \\ IARC: The International Agency for Research on Cancer \\ KEGG: Kyoto Encyclopedia of Genes and Genomes \\ miRNA: MicroRNA \\ mRNA: Messenger RNA \\ MAPK: Mitogen-activated protein kinase \\ RISC: $\quad$ RNA-induced silencing complex \\ SMAD: Small mothers against decapentaplegic \\ TPM: Transcripts per million clean tags.
}

\section{Conflict of Interests}

The authors have declared that no conflict of interests exists.

\section{Authors' Contribution}

Qun Liu and Zhiheng Zhou conceived and designed the research. Chanjiao Zheng and Huanyu Shen were responsible for literature searching and data extraction. Zhiheng Zhou was responsible for the quality assessment of studies. Qun Liu wrote the paper. Zhiheng Zhou and Yixiong Lei revised the paper.

\section{Acknowledgments}

The authors thank the officers and the organization of the field work for their support and assistance in the coordination of this study. This work was supported by the National Natural Science Foundation of China (Yixiong Lei: 81373038 and 81072322; Zhiheng Zhou: 81202236 and 81473001) and Science and Technology Planning Project of Guangdong Province, China (Yixiong Lei: 2013B021800095 and Zhiheng Zhou: 2013B021800093).

\section{References}

[1] W. Swaddiwudhipong, P. Mahasakpan, T. Funkhiew, and P. Limpatanachote, "Changes in cadmium exposure among persons living in cadmium-contaminated areas in Northwestern Thailand: a five-year follow-up," Journal of the Medical Association of Thailand, vol. 93, no. 10, pp. 1217-1222, 2010.

[2] M. P. Waalkes, "Cadmium carcinogenesis," Mutation ResearchFundamental and Molecular Mechanisms of Mutagenesis, vol. 533, no. 1-2, pp. 107-120, 2003.

[3] S. Satarug, J. R. Baker, S. Urbenjapol et al., "A global perspective on cadmium pollution and toxicity in non-occupationally exposed population," Toxicology Letters, vol. 137, no. 1-2, pp. 6583, 2003.

[4] A. Koyu, A. Gokcimen, F. Ozguner, D. S. Bayram, and A. Kocak, "Evaluation of the effects of cadmium on rat liver," Molecular and Cellular Biochemistry, vol. 284, no. 1-2, pp. 81-85, 2006.

[5] G. Nordberg, T. Jin, A. Bernard et al., "Low bone density and renal dysfunction following environmental cadmium exposure in China," Ambio, vol. 31, no. 6, pp. 478-481, 2002.

[6] J. Schöpfer, G. Drasch, and G. N. Schrauzer, "Selenium and cadmium levels and ratios in prostates, livers, and kidneys of nonsmokers and smokers," Biological Trace Element Research, vol. 134, no. 2, pp. 180-187, 2010.

[7] J. Huff, R. M. Lunn, M. P. Waalkes, L. Tomatis, and P. F. Infante, "Cadmium-induced cancers in animals and in humans," International Journal of Occupational and Environmental Health, vol. 13, no. 2, pp. 202-212, 2007.

[8] The International Agency for Research on Cancer, Beryllium, Cadmium, Mercury, and Exposures in the Glass Manufacturing Industry. Working Group Views and Expert Opinions, Lyon, 9-16 February 1993, vol. 58 of IARC Monographs on the Evaluation of Carcinogenic Risks to Humans, World Health Organization, 1993.

[9] Z.-H. Zhou, Y.-X. Lei, and C.-X. Wang, "Analysis of aberrant methylation in DNA repair genes during malignant transformation of human bronchial epithelial cells induced by cadmium," Toxicological Sciences, vol. 125, no. 2, Article ID kfr320, pp. 412417, 2012.

[10] A. Arita and M. Costa, "Epigenetics in metal carcinogenesis: nickel, arsenic, chromium and cadmium," Metallomics: Integrated Biometal Science, vol. 1, no. 3, pp. 222-228, 2009.

[11] B. Wang, Y. Li, C. Shao, Y. Tan, and L. Cai, "Cadmium and its epigenetic effects," Current Medicinal Chemistry, vol. 19, no. 16, pp. 2611-2620, 2012.

[12] Y.-X. Lei, L. Wei, M. Wang, G.-R. Wu, and M. Li, "Malignant transformation and abnormal expression of eukaryotic initiation factor in bronchial epithelial cells induced by cadmium chloride," Biomedical and Environmental Sciences, vol. 21, no. 4, pp. 332-338, 2008.

[13] B. Sen, B. Mahadevan, and D. M. DeMarini, "Transcriptional responses to complex mixtures-a review," Mutation ResearchReviews in Mutation Research, vol. 636, no. 1-3, pp. 144-177, 2007.

[14] A. B. Mendoza-Soto, F. Sánchez, and G. Hernández, "MicroRNAs as regulators in plant metal toxicity response," Frontiers in Plant Science, vol. 3, article 105, 2012.

[15] M. W. Jones-Rhoades, D. P. Bartel, and B. Bartel, "MicroRNAs and their regulatory roles in plants," Annual Review of Plant Biology, vol. 57, pp. 19-53, 2006. 
[16] S. K. Singh, M. P. Bhadra, H. J. Girschick, and U. Bhadra, "MicroRNAs-micro in size but macro in function," The FEBS Journal, vol. 275, no. 20, pp. 4929-4944, 2008.

[17] D. P. Bartel, "MicroRNAs: genomics, biogenesis, mechanism, and function," Cell, vol. 116, no. 2, pp. 281-297, 2004.

[18] R. Schwab, J. F. Palatnik, M. Riester, C. Schommer, M. Schmid, and D. Weigel, "Specific effects of microRNAs on the plant transcriptome," Developmental Cell, vol. 8, no. 4, pp. 517-527, 2005.

[19] S. Choudhuri, "Small noncoding RNAs: biogenesis, function, and emerging significance in toxicology," Journal of Biochemical and Molecular Toxicology, vol. 24, no. 3, pp. 195-216, 2010.

[20] J. Krol, I. Loedige, and W. Filipowicz, "The widespread regulation of microRNA biogenesis, function and decay," Nature Reviews Genetics, vol. 11, no. 9, pp. 597-610, 2010.

[21] Y. Huang, X. J. Shen, Q. Zou, and Q. L. Zhao, "Biological functions of microRNAs," Bioorganicheskaia Khimiia, vol. 36, no. 6, pp. 747-752, 2010.

[22] A. Esquela-Kerscher and F. J. Slack, "Oncomirs-microRNAs with a role in cancer," Nature Reviews Cancer, vol. 6, no. 4, pp. 259-269, 2006.

[23] D. P. Bartel, "MicroRNAs: target recognition and regulatory functions," Cell, vol. 136, no. 2, pp. 215-233, 2009.

[24] X. Fan and L. Kurgan, "Comprehensive overview and assessment of computational prediction of microRNA targets in animals," Briefings in Bioinformatics, 2014.

[25] B. Zhang, X. Pan, Q. Wang, G. P. Cobb, and T. A. Anderson, "Computational identification of microRNAs and their targets," Computational Biology and Chemistry, vol. 30, no. 6, pp. 395407, 2006.

[26] I. Kovalchuk, V. Titov, B. Hohn, and O. Kovalchuk, "Transcriptome profiling reveals similarities and differences in plant responses to cadmium and lead," Mutation Research, vol. 570, no. 2, pp. 149-161, 2005.

[27] Z. S. Zhou, J. B. Song, and Z. M. Yang, "Genome-wide identification of Brassica napus microRNAs and their targets in response to cadmium," Journal of Experimental Botany, vol. 63, no. 12, pp. 4597-4613, 2012.

[28] X. Fang, Y. Zhao, Q. Ma et al., "Identification and comparative analysis of cadmium tolerance-associated miRNAs and their targets in two soybean genotypes," PLoS ONE, vol. 8, no. 12, Article ID e81471, 2013.

[29] L. W. Zhang, J. B. Song, X. X. Shu, Y. Zhang, and Z. M. Yang, "MiR395 is involved in detoxification of cadmium in Brassica napus," Journal of Hazardous Materials, vol. 250-251, pp. 204211, 2013.

[30] Y. Ding, A. Qu, S. Gong, S. Huang, B. Lv, and C. Zhu, "Molecular identification and analysis of Cd-responsive microRNAs in rice," Journal of Agricultural and Food Chemistry, vol. 61, no. 47, pp. 11668-11675, 2013.

[31] Y. Ding, Z. Chen, and C. Zhu, "Microarray-based analysis of cadmium-responsive microRNAs in rice (Oryza sativa)," Journal of Experimental Botany, vol. 62, no. 10, pp. 3563-3573, 2011.

[32] M. Martínez-Pacheco, A. Hidalgo-Miranda, S. RomeroCórdoba, M. Valverde, and E. Rojas, "MRNA and miRNA expression patterns associated to pathways linked to metal mixture health effects," Gene, vol. 533, no. 2, pp. 508-514, 2014.

[33] M. Kanehisa, M. Araki, S. Goto et al., "KEGG for linking genomes to life and the environment," Nucleic Acids Research, vol. 36, no. 1, pp. D480-D484, 2008.
[34] M. Kanehisa and S. Goto, "KEGG: kyoto encyclopedia of genes and genomes," Nucleic Acids Research, vol. 28, no. 1, pp. 27-30, 2000 .

[35] E. Altermann and T. R. Klaenhammer, "PathwayVoyager: pathway mapping using the Kyoto Encyclopedia of Genes and Genomes (KEGG) database," BMC Genomics, vol. 6, article 60, 2005.

[36] K. J. Livak and T. D. Schmittgen, "Analysis of relative gene expression data using real-time quantitative PCR and the $2^{-\Delta \Delta C T}$ method," Methods, vol. 25, no. 4, pp. 402-408, 2001.

[37] J. Luevano and C. Damodaran, "A review of molecular events of cadmium-induced carcinogenesis," Journal of Environmental Pathology, Toxicology and Oncology, vol. 33, no. 3, pp. 183-194, 2014.

[38] J. Chmielowska-Bak, J. Gzyl, R. Rucinska-Sobkowiak, M. Arasimowicz-Jelonek, and J. Deckert, "The new insights into cadmium sensing," Frontiers in Plant Science, vol. 5, article 245, 2014.

[39] G. A. Calin, C. Sevignani, C. D. Dumitru et al., "Human microRNA genes are frequently located at fragile sites and genomic regions involved in cancers," Proceedings of the National Academy of Sciences of the United States of America, vol. 101, no. 9, pp. 2999-3004, 2004.

[40] M. Lagos-Quintana, R. Rauhut, W. Lendeckel, and T. Tuschl, "Identification of novel genes coding for small expressed RNAs," Science, vol. 294, no. 5543, pp. 853-858, 2001.

[41] Y. Lee, K. Jeon, J.-T. Lee, S. Kim, and V. N. Kim, "MicroRNA maturation: stepwise processing and subcellular localization," The EMBO Journal, vol. 21, no. 17, pp. 4663-4670, 2002.

[42] X. Cai, C. H. Hagedorn, and B. R. Cullen, "Human microRNAs are processed from capped, polyadenylated transcripts that can also function as mRNAs," RNA, vol. 10, no. 12, pp. 1957-1966, 2004.

[43] Y. Lee, M. Kim, J. Han et al., "MicroRNA genes are transcribed by RNA polymerase II," The EMBO Journal, vol. 23, no. 20, pp. 4051-4060, 2004. 

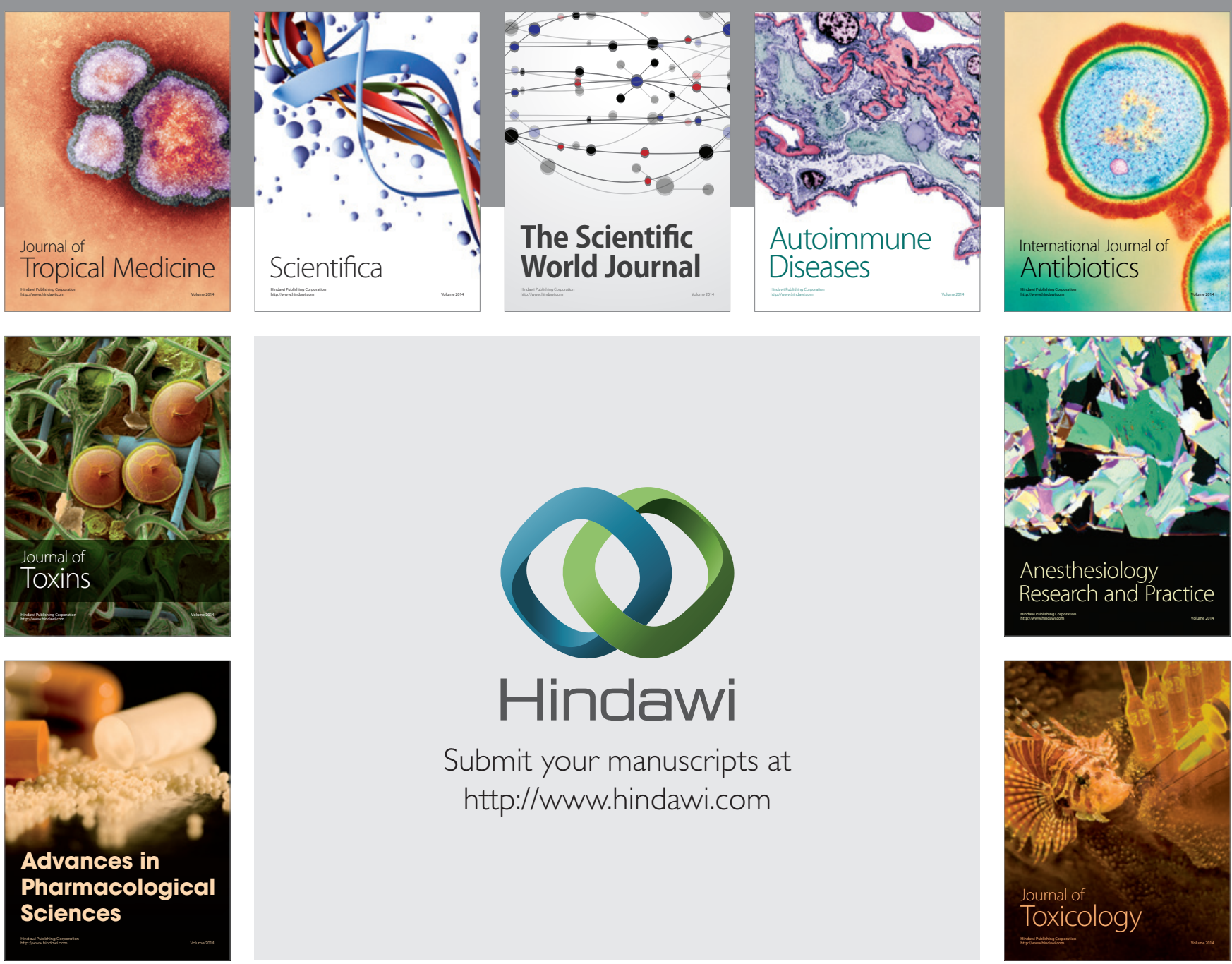

\section{Hindawi}

Submit your manuscripts at

http://www.hindawi.com
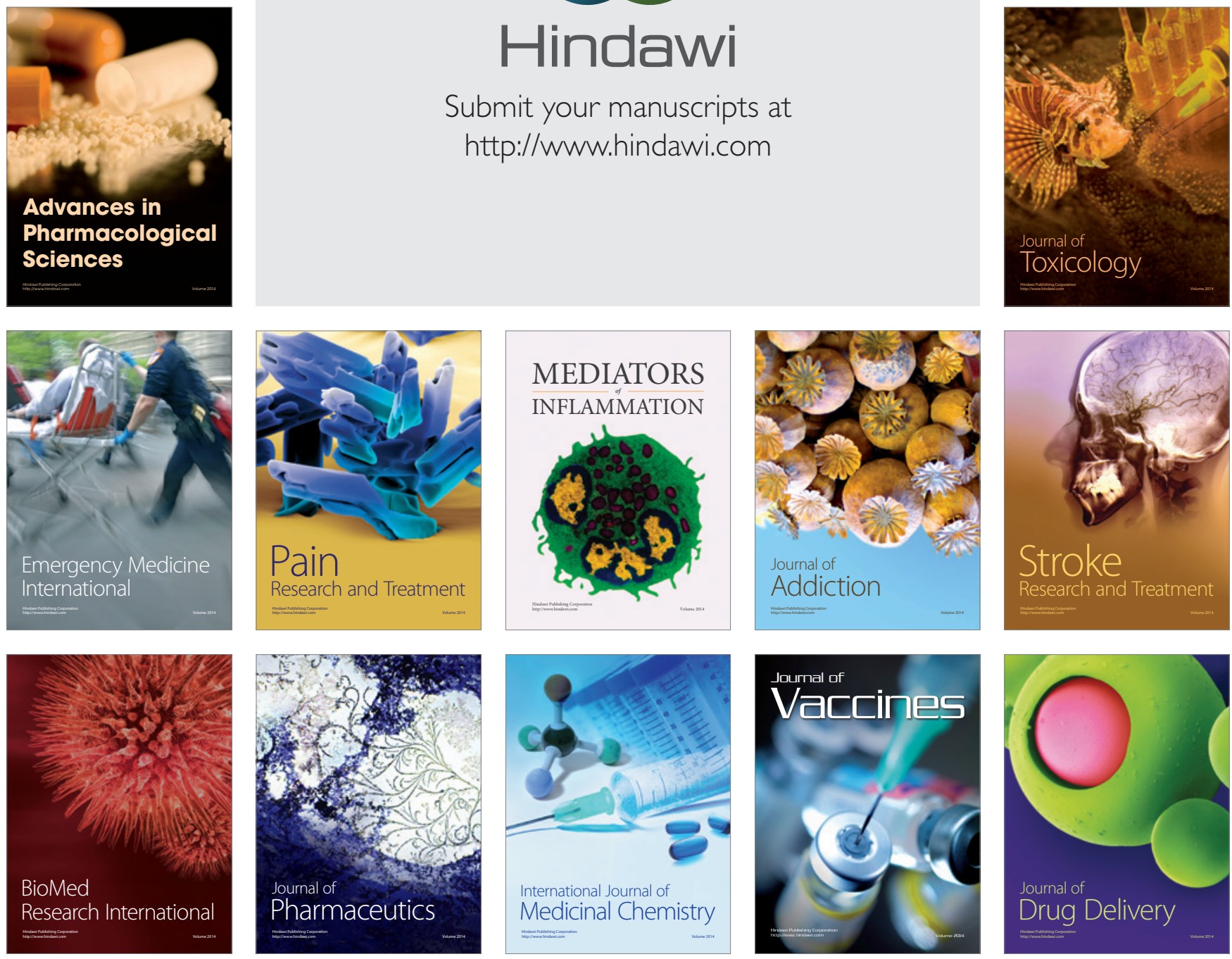